\title{
A Review on Efficacy of Siddha Medicinal Herbs as Antiviral Drugs against COVID-19
}

\author{
Review Article
}

\section{Muralidass S D ${ }^{*}$, Shree Devi M S²}

1. Siddha Physician, Arumbakkam, Chennai.

2. Research Officer (Siddha), Siddha Central Research Institute, Chennai.

\begin{abstract}
In a very short time, the Coronavirus disease (COVID-19) caused by the SARS-CoV-2 virus, has become a global pandemic. It has infected about 29,155,581 patients and brought forth death rate about 926,544 among 216 countries as recorded by World Health Organization on 15th September 2020. At present, there is no specific and direct treatment or vaccine for this infectious disease. No therapeutic options available to cure the infection in till date which increases the mortality rate across the countries. Currently there is no antiviral treatments have been approved, several approaches have been proposed in western medicine, Chinese medicine, CAM, AYUSH and other systems. Siddha Herbal medicines are achieving high attention because of the extensive therapeutics like potent anti-microbial, immunomodulatory, anti-inflammatory, and antioxidant properties. Ministry of AYUSH, Govt. of India recommended 12 Siddha herbals (Inji - Zingiber officinale, Thulasi - Ocimum sanctum, Milaku - Piper nigrum, Karunjeeragam Nigella sativa, Keezhanelli - Phyllanthus niruri, Adhimathuram - Glycyrrhiza glabra, Vellaippoondu - Allium sativum, Citramutti - Sida cardifolia, Seenthil - Tinospora cordifolia, Manjal - Curcuma longa, Elumitchai - Citrus lemon and Vembu - Azadirachta indica) for the prevention by enhancing immunity as well as for the management of this infectious disease in its recent official publication 'Guidelines for Siddha Practitioners for COVID 19'. This is the common advisory and the anti-viral activity of these herbals against COVID 19 is not established yet. The current situation demands proper herbal drug utilization to prepare the population immunity to fight against the infection. The present review spotlight on the literature review and the review of pharmacological actions of these herbals.
\end{abstract}

Key Words: Siddha Medicine, Antiviral herbs, Phytochemicals, Immunity boosting, SARS-CoV-2, AYUSH.

\section{Introduction}

Coronavirus Disease 2019 (COVID-19) is defined as illness caused by a novel coronavirus now called Severe Acute Respiratory Syndrome Coronavirus 2 (SARS-CoV-2; formerly called 2019-nCoV), which was first identified amid an outbreak of respiratory illness cases in Wuhan City, Hubei Province, China (1). Initially it was reported to the WHO on $31^{\text {st }}$ December 2019. WHO declared the COVID - 19 outbreaks a global health emergency on $30^{\text {th }}$ January $2020(3,4)$ and global pandemic on 11 th March 2020 (5). This global pandemic in a short time has infected about 29,155,581 patients and brought forth death rate about 926,544 among 216 countries as mentioned by World Health Organization on $15^{\text {th }}$ September 2020 (6). The main symptoms include: Fever or chills, Cough, Shortness of breath, Trouble breathing, Fatigue, Body aches, Headache, Running or stuffy nose, Sore throat, Loss of smell or taste,

\section{* Corresponding Author:}

\section{Muralidass SD}

Siddha Physician,

Arumbakkam,

Chennai - 600106

Tamil Nadu. India.

Email Id: drmuralinis@gmail.com
Nausea, Diarrhoea (2). Presentations of COVID-19 have ranged from asymptomatic/mild symptoms to severe illness and mortality. Symptoms may develop two days to two weeks following exposure to the virus (7). Currently, there is no specific treatment or vaccine for fighting against this infectious disease and scientists agree that possible therapeutic may arise through drug repositioning. Herbal medicines are achieving high attention because of the extensive therapeutics like potent anti-viral, immuno-modulatory, antiinflammatory, and antioxidant properties.

Siddha System of Medicine (SSM) is an ancient Medical system which is in Tamil language flourished more in southern part of India. This medical system has its own school of terminologies for naming the diseases, signs and symptoms, diagnostic methods, pharmacological and non-pharmacological therapies in the Tamil language. In SSM, Epidemics/Pandemics are mentioned as "Uzhi Noi". In general, they are classified under "Kollai Noikal" (Communicable Diseases) which are most commonly occurred the time of "Ayana Santhi" months (Means end a month of Uthara Ayanam \& Thatchana Ayanam), fall on Aadi and Maargazhi month in Tamil Calendar. It is believed that in those days, the immunity of human beings will be low based on Mukkutram Theory (Three humoural theories - Vaatham, Piththam and Kabam) (8). 
The symptoms present in COVID-19 are compared with the disease 'Kabasuram' mentioned in SSM. As per the Siddha literature tamil quote 'ver paaru thazhai paaru minjinakkaal parpa chenduram paarae', herbal medicines are the first choice of drugs for all diseases in SSM, if satisfied result not occurs with herbal ingredients, higher order medicines prepared from Metal and Mineral, and from Marine products are prescribed. So, this emphasizes the usage and importance of Herbals in SSM (9).

Ministry of AYUSH, Govt. of India recently released its official publication 'Guidelines for Siddha Practitioners for COVID 19' on May 31, 2020. This guideline recommended Siddha herbs for the prevention by enhancing immunity as well as for the management of this infectious disease which are being prescribed for acute viral and bacterial respiratory diseases and other chronic respiratory diseases in SSM from ancient times.
These Anti-viral Siddha herbs are tabulated in Table I. This guideline is the common advisory for Siddha Practitioners and the anti-viral activity of these herbals against COVID 19 are not established yet (8). So, the author made attempt to discuss the literature review and the review of pharmacological actions of these herbals in this article.

\section{Materials and Methods \\ Data source}

The relevant literature is referred and collected from Siddha text and contemporary literature. Literature related to title is referred from all Siddha reliable journals and other herbal related journals. Published research works on individual herbs have been collected based on their therapeutic actions from net resources and reviewed.

Table I -Anti-Viral Siddha Herbs and their therapeutic uses in $\operatorname{SSM}(8,10)$

\begin{tabular}{|c|c|c|c|}
\hline S.No & Common Name & Botanical Name & Usage \\
\hline 1 & Inji & Zingiber officinale ROSC. & $\begin{array}{l}\text { 1. Inji Surasam - Herbal water infusion made up of } \\
\text { half teaspoon of Chukku (Dried Ginger) boiled in two } \\
\text { liters of water can be used as drinking water }-10 \mathrm{ml} \\
\text { Once a day } \\
\text { 2. Inji Thenooral - dipped and processed in honey - } \\
\text { one piece at early morning } \\
\text { 3. Inji Tea (Ginger tea) as hot beverage }\end{array}$ \\
\hline 2 & Thulasi & Ocimum tenuiflorum $\mathrm{L}$. & $\begin{array}{l}\text { 1. Thulasi Kudineer - Prepared as decoction - } 60 \mathrm{ml} \\
\text { Twice a day } \\
\text { 2. Used as Steam inhalation }\end{array}$ \\
\hline 3 & Milagu & Piper nigrum L. & $\begin{array}{l}\text { 1. With Thulasi as Kudineer - Prepared as decoction - } \\
60 \mathrm{ml} \text { Twice a day } \\
\text { 2. A quarter teaspoonful of Manjal (Turmeric) with } \\
\text { one pinch of Milagu (Pepper) is added in boiling milk } \\
\text { and taken at bed time. }\end{array}$ \\
\hline 4 & Karunjeerakam & Nigella sativa $\mathrm{L}$. & $\begin{array}{l}\text { Karunjeeraka Chooranam - Prepared as powder - } \\
1 \text { gm Twice a day }\end{array}$ \\
\hline 5 & Keezhanelli & $\begin{array}{l}\text { Phyllanthus amarus SCHUM. } \\
\& \text { THENN. }\end{array}$ & $\begin{array}{l}\text { Keezhanelli Samoolam (whole plant) - Prepared as } \\
\text { powder - 2gm Twice a day }\end{array}$ \\
\hline 6 & Athimadhuram & Glycyrrhiza glabra L. & $\begin{array}{l}\text { 1. Athimadhura Chooranam - Prepared as powder - } 1 \\
\text { gm Twice a day } \\
\text { 2. Adhimadhuram (Licorice) Tea - taken } \\
\text { as hot beverage }\end{array}$ \\
\hline 7 & Vellaipoondu & Allium sativum L. & $\begin{array}{l}\text { Poonduthaen - Juice of Poondu (Garlic) is processed } \\
\text { with honey by heating process then used as externally } \\
\text { to apply on tongue, tonsils and oral cavity }\end{array}$ \\
\hline 8 & Citra mutti & Sida cordifolia $\mathrm{L}$. & $\begin{array}{l}\text { Citramutti Kudineer - Prepared as decoction - } 30 \mathrm{ml} \\
\text { Twice a day }\end{array}$ \\
\hline 9 & Seenthil & $\begin{array}{l}\text { Tinospora cordifolia (WILLD.) } \\
\text { HOOK.F. \& THOMS. }\end{array}$ & $\begin{array}{l}\text { Seenthil Chooranam - Prepared as powder - 1gm } \\
\text { Twice a day }\end{array}$ \\
\hline 10 & Manjal & Curcuma longa L. & $\begin{array}{l}\text { 1. A quarter teaspoonful of Manjal (Turmeric) with } \\
\text { one pinch of Milagu (Pepper) is added in boiling milk } \\
\text { and taken at bed time. } \\
\text { 2. Water boiled with a pinch of Salt and Manjal } \\
\text { (Turmeric) should be used for gargling not less than } 3 \\
\text { times }\end{array}$ \\
\hline 11 & Elumitchai & Citrus limon (L.) BURM.F. & Elumitchai (Lemon) Tea - taken as hot beverage \\
\hline 12 & Vembu & Azadirachta indica A. JUSS. & $\begin{array}{l}\text { Vembu (Neem) leaves fumigation is advocated for } \\
\text { Environmental sanitation }\end{array}$ \\
\hline
\end{tabular}


Table II - Anti-Viral Siddha Herbs and its Pharmacological properties (11)

\begin{tabular}{l|l|l|l|l|} 
S.No & Common Name & Part used & Actions & Indications \\
\hline
\end{tabular}

\begin{tabular}{|c|c|c|c|c|}
\hline 1 & $\begin{array}{l}\text { Inji-Zingiber } \\
\text { officinale }\end{array}$ & Rhizome & $\begin{array}{l}\text { Stimulant, Stomachic, } \\
\text { Carminative }\end{array}$ & $\begin{array}{l}\text { Dyspepsia, Heartburns, Flatus, } \\
\text { Cough, Asthma, Diarrhea, Sinusitis, } \\
\text { Peptic Ulcer, Anemia, Fever }\end{array}$ \\
\hline 2 & $\begin{array}{l}\text { Thulasi - Ocimum } \\
\text { sanctum }\end{array}$ & Leaf, Seed & $\begin{array}{l}\text { Stimulant, Expectorant, } \\
\text { Demulcent, Diaphoretic }\end{array}$ & $\begin{array}{l}\text { Psychiatry diseases, Sinusitis, Fever, } \\
\text { Vatha and Kaba diseases }\end{array}$ \\
\hline
\end{tabular}

$3 \quad$ Milagu-Piper $\quad$ Seed nigrum

$4 \quad$ Karunjeerakam - $\quad$ Seed Nigella sativa

5

Keezhanelli Phyllanthus niruri

Whole plant

6 Athimadhuram - $\quad$ Root Glycyrrhiza glabra

7

Vellaipoondu Allium sativum

8

9

Citra mutti - Sida cordifolia

9

Seenthil -

Tinospora cordifolia

0 Manjal-Curcuma longa

$1 \quad$ Elumitchai Citrus limonia

Vembu Azadirachta indica

Whole plant

Rhizome fruit, Fruit oil

\section{Leaf, Stem} bark, Root

\section{Rhizome}

Emollient

Acrid, Carminative, Antiperiodic, Rubefacient, Stimulant, Resolvent, Antivatha, Antidote

Carminative, Diuretic, E m m e n a o g u e, $\mathrm{G}$ a 1 a c t o g o g u e, Anthelmintic, Stomachic, Parasiticide, Emollient

\section{Deobstruent, Diuretic,} Astringent, Cooling

Tonic, Emollient, Laxative, Mild expectorant.

Stimulant, Stomachic, Carminative, Tonic, Alterative, Expectorant, Anthelmintic, Diuretic

$\begin{array}{ll}\text { Stem } & \text { Alterative, Stimulant, } \\ \text { Demulcent, Antiperiodic }\end{array}$

Leaf, Unripe juice, volatile bark, Flower, Unripe fruit, Fruit, Seed, Seed oil, Pisin

\section{Stimulant, Carminative, Hepatic tonic}

Refrigerant, Rubefacient, Carminative

Anthelmintic, Stimulant, Discutient
Fever with chill, Anemia, Phlegm, Diarrhea, Gastritis, Flatulence, Anorexia, Hemorrhoids, Cough, Paralysis, Earache, Indigestion, Jaundice

Eczema, Skin ulcer, Diseases of the head, Eye diseases, Scabies, Flatulence, Gastric ulcer, Chest pain, Cough, Vomiting, Swelling, Jaundice

Abdominal discomfort, Eye diseases, Bleeding diarrhea, Diabetes, Jaundice, Fever caused by abnormalities in seven physical constituents, Chronic syphilitic ulcer, Dryness of the tongue, Thirst

Liver disorders, Fever, Phlegmatic affections, Cough, Headache, Eye diseases, Psychiatric illness, Diseases of bone and as Antidote

Small boils, Deafness, Chronic cough, Asthma, Worm infestations, Thiridosha diseases, Vatha diseases, Headache by imbalanced kabam, Heaviness of the head, Dysentery, Hemorrhoids

Fever associated with bone disorders, Pitha diseases, Thirst, Diarrhea, Flatulence, Gastritis, Fever by Vatha imbalance

Fever, Diabetes, Skin diseases, Diarrhea, Hypertension, Purpura

Vomiting, Thiridosham imbalance, Headache, Heaviness of the head, Running nose, Five types of fits, Swelling, Skin ulcer

Thiridosham Imbalance, Pricking Pain, Vomiting, Gastric Ulcer, Pitha Diseases, Psychiatry Diseases, Giddiness, Nausea, Thirst, Eye diseases, Earache, paronychia

Vomiting, Dyspepsia, Thirst, Skin diseases. Fever, Convalescence after fever, Indigestion, Vatha diseases, Peptic ulcer, Worm infestation 
Muralidass SD et.al., A Review on Efficacy of Siddha Medicinal Herbs as Antiviral Drugs against COVID-19

\section{Table III - Anti-Viral Siddha Herbs and its Phytochemical properties}

\section{S.No Common Name Phytochemical}

$1 \quad$ Inji-

Zingiber officinale

$2 \quad$ Thulasi-

Ocimum sanctum

$3 \quad$ Milagu-

Piper nigrum

4 Karunjeerakam -

Nigella sativa

$5 \quad$ Keezhanelli -

Phyllanthus niruri

6 Athimadhuram Glycyrrhiza glabra

7 VellaipoonduAllium sativum

$8 \quad$ Citra muttiSida cordifolia

9 Seenthil-

Tinospora cordifolia
Cardiac glycosides, Alkaloids, Saponins, Tannins, Flavonoids, Sesquiterpenoids, Monoterpenes, Bisapolene, Zingiberene, and Zingiberol (Connel D), 6-Shogaol, Gingerols, Zingerone, Paradol, Zingerone, Geraniol, Gingerols, Gingerdiols, Gingerdiones, and Dehydrogingerdiones $(12,13)$

The leaves contain $0.7 \%$ volatile oil comprising about $71 \%$ Eugenol and $20 \%$ Methyl eugenol. The oil also contains Carvacrol and Sesquiterpine hydrocarbon caryophyllene. Fresh leaves and stem extract yielded some phenolic compounds (antioxidants) such as Cirsilineol, Circimaritin, Isothymusin, Apigenin and Rosameric acid, Ursolic acid, Apigenin, Luteolin, Apigenin-7-O-glucuronide, Luteolin-7-O glucuronide, Orientin and Molludistin have also been isolated from the leaf extract. It also contains a number of sesquiterpenes and monoterpenes viz., Bornyl acetate, -Elemene, Neral, - and -Pinenes, Camphene, Campesterol, Cholesterol, Stigmasterol and -Sitosterol $(14,15)$

Propanedioic acid, Dimethyl ester, Bicyclo heptane,3-Carene, Cyclohexene, Piperine, Ursodeoxycholic acid, Piperidine, Piperettine and Piperanine, Stigmasterol (16) Seeds contain Thymoquinone (30\%-48\%), Thymohydroquinone, Dithymoquinone, $\mathrm{p}$ Cymene (7\%- 15\%), Carvacrol (6\%-12\%), 4-Terpineol (2\%-7\%), t-Anethol (1\%-4\%), Sesquiterpene longifolene (1\%-8\%) $\alpha$-Pinene and Thymol. The seeds are also containing good amount of various vitamins and minerals like $\mathrm{Cu}, \mathrm{P}, \mathrm{Zn}$ and $\mathrm{Fe}$. Root and shoot are reported to contain Vanillic acid. The seeds reported to contain a fatty oil rich in unsaturated fatty acids, mainly Linoleic acid (50-60\%), Oleic acid (20\%), Eicodadienoic acid (3\%) and Dihomolinoleic acid (10\%). (17)

Rutin, Quercetin, Quercitrin, Astragalin, Gallocatechin, Nirurin, Niruriflavone, Limonene, p-Cymene, Lupeol, Ellagic acid, Methyl brevifolincarboxylate, Phyllanthin and Hypophyllanthin, Niranthin, Nirtetralin, Phyltetralin and Lintetralin, Repandusinic acid, Norsecurinine, Diosgenin. (18)

Triterpenoid saponins (4-20\%), mostly Glycyrrhizin, a mixture of potassium and calcium salts of 18ß-glycyrrhizic acid, triterpenes included Liquiritic acid, Glycyrretol, Glabrolide, isoglaborlide and Liquorice acid. 18ß-glycyrrhizic acid (3O-(2-O- $\beta$-d- glucopyranuronosyl- $\alpha$-d-glucopyranurosyl)-3- $\beta$-hydroxy-11-

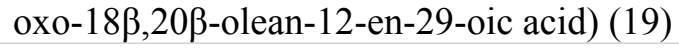

alliin, vitamin $\mathrm{C}$, gallic acid, catechin hydrate, fructose, glucose, adenosine, arginine, ascorbic-acid, calcium, magnesium, potassium, quercetin, tryptophan and tyrosinase. (20)

Large amount of Ephedrine, Sterculic, Malvalic and Coronaric acid along with other fatty acids, Pseudoephedrine, Saponine, Choline pseudoephedrine, Betaphenethylamine, Vasicine, Hypaphorine, Ecdysterone and related indole alkaloids, Palmitic, Stearic and $\beta$ - sitosterol, Hexacosanoic acids, 6-phenyl ethyl amine, Carboxylated tryptomines, Qunazoline, Hypaphorine, Vasicinol (21)

Stem and root part contain alkaloids as active constituents. These are Tembetarine, Choline, Magnoflorine, Berberine, Tinosporin, Isocolumbin, Palmetine, Jatrorrhizine, Aporphine alkaloids, Tetrahydropalmatine. Whole plant of contain Furanolactone, Diterpenoid Lactones, Cleodrane derivatives, Columbin tinosporides, Tinosporin, Jateorine. Shoot part of contains Steroids (B-sitosterol, $\delta$-sitosterol, $20 \beta$ hydroxyecdysone, giloinsterol, Makisterone A, Ecdysterone). Stem part contain Glycosides. Their active constituents are 18-norcleodrane glucoside, Tinocordifolioside, Cordioside, Cordifolioside A, B, C, D and E, Furanoid diterpine glucoside, Syringin, Pregnane glycoside Syringing-apiosylglycoside, palmatosides, The other parts contain active constituents such as Jatrorrhizine, Tinosporic acid, 3, (a, 4-di hydroxy-3-methoxy-benzyl)-4-(4- hydroxy-3-methoxy-benzyl) Tetrahydrofuran, N-trans-feruloyltyramine as diacetate, Giloin. (22) 
10 Manjal -

Curcuma longa

11 Elumitchai-

Citrus limonia

12

Vembu -

Azadirachta indica 1,8-cineole, 2-bornanol, 2-hydroxymethyl-anthraquinone, 4-hydroxybisabola, 10diene-9-one; 4-methoxy-5- hydroxybiosabola; 4-hydroxy-cinnamoyl- (Feruloyl)methane, Alpha-atlantone, Alphapinene, Alphaterpineol, Ar-turmerone, Arabinose. Ascorbic-acid, Ash, Azulene, Betacarotene, Beta-pinene, Beta sesquiphellandrene, Bis-(Para-hydroxycinnamoyl)-methane. Bis-desmethoxycurcumin, Bisabolene, Bixin, Borneol, Boron, Caffeic-acid, Calcium, Caprylic-acid, Caryophyllene, Chromium, Cineole, Cinnamic-acid, Cuminyl-alcohol, Curcumene, Curcumenol, Curcumin, Curdione, Cobalt, Copper. Eugenol, Epiprocurcumenol; Eucalyptol; Eugenol; Feruloyl-p-coumaroyl-methane, Gamma-atlantone, Germacrone, Germacrone13-al; Guaiacol, Isoborneol, L-alphacurcumene. L-beta-curcumene, Limonene, Manganese, Monodesmethoxycurcumin, Niacin, Nickel, norbixin; Ocoumaric-acid, P-coumaric-acid, P-methoxycinnamic-acid, Pcymene, Ptolymethylcarbinol, Phosphorus, Protocatechuic-acid, Procurcumadiol. Acidic polysaccharides: utonan A, B, C, D. Volatile Oil (4.2\%), Other chemicals compound are copper/zinc, campesterol, stigmasterol, betasitosterol, cholesterol, fatty acids and metallic elements potassium, sodium, magnesium, calcium, manganese, iron. (23) Citronellal (29.31\%), Limonene (17.59\%), (E)-citral (12.71\%), 1,6-octadien-3-ol,3,7dimethyl (10.91\%), biocyclo [3.1.0] hexane, 4-mehylene-1-(1-methyl) (8.80\%), 6octen-1-ol,3,7-dimethl (7.95\%), 2,6-octadien-1-ol,3,7-dimethyl-acetate (Z) (6.29\%), 1,3-cyclohexadiene,5-(1,5-dimethyl-4-hexenyl)-2-methyl, [S(R,S)] (2.81\%), cyclohexene,3-(1,5-dimethyl-4-hexenyl)-6-methylene-, [S-(R,S)](1.64\%), bezene,1(1,5-dimethyl-4-hexenyl)- 4-methyl (1.10\%) and cyclohexene,1-methyl-4-(5methyl-1-methyl-1-methylene-4-hexenyl)-,(s) (0.88\%)

Flavanones (Eriocitrin 16.7\%, Hesperidin 20.5\%); Flavones (6,8-di-CGluApigenin1.17\%，6,8-di-C-Glu-Diosmetin 4.95\%，7-O-Rut-Luteolin 3.93\%, Diosmin 3.12\%), Aglycones (Luteolin 0.08\%)].

Coumarins and Psoralens: (Bergapten, Bergamottin, Byakangelicin, Citropten, Imperatorin, Isoimperatorin, Isopimpinellin, phellopterin, Prangol, Scoparon, Scopoletin, Umbelliferone, Umbelliprenin, Xanthyletin). Carotenoids: (Phytofluene, $\beta$-carotene, Cryptoxanthin, Violaxanthin, Auroxanthin). (24)

Azadirachtin and the others are Nimbolinin, Nimbin, Nimbidin, Nimbidol, Sodium nimbinate, Gedunin, Salannin, and Quercetin. Leaves contain ingredients such as Nimbin, Nimbanene, 6-desacetylnimbinene, Nimbandiol, Nimbolide, Ascorbic acid, n-hexacosanol and amino acid, 7-desacetyl-7-benzoylazadiradione, 7-desacetyl-7benzoylgedunin, 17-hydroxyazadiradione, and nimbiol. Quercetin and $\beta$-sitosterol, polyphenolic flavonoids, were purified from neem fresh leaves. Seeds hold valuable constituents including Gedunin and Azadirachtin (25)

\section{Discussion and Conclusion}

In this review, mentioned herbals are being prescribed for acute respiratory infectious diseases and other chronic respiratory diseases in Siddha System of Medicine from ancient times. They are also indicated for various ailments. Studies proven the antibacterial, anti-viral and other anti-microbial effects against various microbes of these Siddha herbals. Most of these herbals having anti- pyretic, anti-inflammatory and antioxidant effects which were proved through various studies. Some herbals in guideline have specific effects on Asthma and other respiratory diseases. Moreover, all the herbals are having good effect on Diabetes. And they are prescribed for neurological, cardio vascular, Gastro intestinal, excretory, and Genito urinary diseases and effect in the systems also were proven in research studies.
The Siddha formulations mentioned in the 'Guidelines for Siddha Practitioners for COVID 19' are Kabasura Kudineer Chooranam, Nilavembu Kudineer Chooranam, Nellikkai legiyam, Amukkara Chooranam, Thalisadhi Chooranam, Adhimadura Chooranam, Thippili Rasayanam, Notchi Kudineer Chooranam, Vishasura Kudineer Chooranam. They are highly used in the Covid care centers of South India indicating possible effects at present and some clinical trials are going on such siddha medicines. Most of these 12 herbals are as one of the ingredients of the abovementioned preparations.

Anti-viral activity of these herbals against COVID 19 is not established yet, anyway the Pharmacological review these herbals indicates that they may be suitable for the treatment of COVID 19 disease even with co morbidity conditions because all 
the herbals are having good effects on Respiratory system as well as other systems. Proper combination of herbs for activity study against current viral infection may help to prepare the healthcare to prepare against any health-related issues. The creation of awareness is very important among all people regarding prevention of Covid-19 infection.

\section{Conflict of interest} Nil

\section{References}

1. https://www.cdc.gov/coronavirus/2019-ncov/about/ index.html\# dated 26.01.2020 time 17:24 IST

2. https://www.webmd.com/lung/coronavirus\# dated 11.08.2020 time 08:38 IST

3. https://www.medscape.com/viewarticle/924596\# dated 30.01.2020 time 11:05 IST

4. https://nyti.ms/2RER70M\# dated 30.01.2020 time 09:05 IST

5. https://www.nytimes.com/2020/03/11/world/ coronavirus-news.html\#link-682e5b06\# dated 11.03.2020 time 19:15 IST

6. https://www.who.int/emergencies/diseases/novelcoronavirus-2019\# dated 08.09.2020 time 20:58 IST

7. https://www.cdc.gov/coronavirus/2019-ncov/about/ symptoms.html\# dated 26.01.2020 time 07:19 IST

8. Guidelines for Siddha Practitioners for COVID 19. Ministry of AYUSH, Govt of India; 2020. 04-06p.

9. Thiyagarajan R. Gunapadam part 2 Thaathu Jeeva Vaguppu. $2^{\text {nd }}$ edition. Chennai; Directorate of Indian Medicine and Homeopathy; 2006. 56-86p.

10. https://envis.frlht.org/\# dated 24.07.2020 time 06:48 IST

11. Murugesa Muthaliyar KS. Gunapadam part 1 Mooligai Vaguppu. 2nd edition. Chennai; Directorate of Indian Medicine and Homeopathy; 2008. 98-101, 531-534, 760-763, 463-464, 345-347, 13-16, 846-847, 446-447, 454-458, 720-722, 158-160, 853-861p.

12. Fatai Oladunni Balogun, Esther Tayo Adeye Oluwa and Anofi Omotayo Tom Ashafa, Pharmacological Potentials of Ginger, Ginger Cultivation and Its Antimicrobial and Pharmacological Potentials. Haiping Wang, Intech Open. DOI: 10.5772/ intechopen. 88848 .

13. Osabor VN, Bassey FI, Umoh UU, Phytochemical Screening and Quantitative Evaluation of Nutritional Values of Zingiber officinale (Ginger). American Chemical Science Journal. January, 2015; 8(4): 1-6

14. Govind Pandey, Madhuri S, Pharmacological Activities of Ocimum sanctum (Tulsi): A Review. International Journal of Pharmaceutical Sciences
Review and Research. November-December, 2010; 5(1); 61-66

15. Deepika Singh, Prabir K Chaudhuri, A review on phytochemical and pharmacological properties of Holy basil(Ocimum sanctum L.). Industrial Crops \& Products. August, 2018; 118; 367-382

16. Ghaidaa Jihadi Mohammed, Aseel Mohammad Omran, Haider Mashkoor Hussein, Antibacterial and Phytochemical Analysis of Piper nigrum using Gas Chromatography - Mass Spectrum and Fourier-Transform Infrared Spectroscopy. International Journal of Pharmacognosy and Phytochemical Research. June, 2016; 8(6); 977-996

17. Desai SD, Shaik Hussain Saheb, Kusal K Das, Haseena S, Phytochemical Analysis of Nigella sativa and it's Antidiabetic Effect. J. Pharm. Sci. \& Res. August, 2015; 7(8); 527-532

18. Bagalkotkar G, Sagineedu SR, Saad MS, Stanslas J, Phytochemicals from Phyllanthus niruri Linn. and their pharmacological properties: a review. Journal of Pharmacy and Pharmacology. December, 2006; $58 ; 1559-1570$

19. Isbrucker RA, Burdock GA, Risk and safety assessment on the consumption of Licorice root (Glycyrrhiza sp.), its extract and powder as a food ingredient, with emphasis on the pharmacology and toxicology of glycyrrhizin. Regular Toxicol Pharmacol. December, 2006; 46; 167-92

20. Tiwari S, Sirohi B, Shukla A, Bigoniya P, Phytochemical Screening and Diuretic Activity of Allium sativum Steroidal and Triterpenoid Saponin Fraction. Int J Pharm Sci Res. August, 2012; 3(9); 3354-3361

21. Ankit Jain, Shreya Choubey, Singour PK, Rajak H, R.S. Pawar, Sida cordifolia (Linn) - An overview. Journal of Applied Pharmaceutical Science. April, 2011; 01 (02); 23-31

22. Prashant Tiwari, Puravi Nayak, Shakti Ketan Prusty, Pratap Kumar Sahu, Phytochemistry and Pharmacology of Tinospora cordifolia: A Review. Sys Rev Pharm. January-December, 2018; 9(1); 70-78

23. Sayantani Chanda, Ramachandra TV, Phytochemical and Pharmacological Importance of Turmeric (Curcuma longa): A Review. Research \& Reviews: A Journal of Pharmacology. February, 2019; 9(1); 16-23

24. Ali Esmail Al-Snafi, Nutritional value and pharmacological importance of citrus species grown in Iraq. IOSR Journal of Pharmacy. August, 2016; 6(8); 76-108

25. Mohammad A. Alzohairy, Therapeutics Role of Azadirachta indica (Neem) and Their Active Constituents in Diseases Prevention and Treatment. Evid Based Complement Alternat Med. March, 2016; 2016; 7382506. 